\title{
AN EXTENSION OF THE MINKOWSKI DETERMINANT THEOREM
}

\author{
by MARVIN MARCUS AND WILLIAM R. GORDON $\dagger$
}

(Received 21st September 1970)

Minkowski proved the following (for a proof see (4)): if $A$ and $B$ are $n \times n$ positive semi-definite hermitian matrices then

$$
(\operatorname{det}(A+B))^{1 / n} \geqq(\operatorname{det} A)^{1 / n}+(\operatorname{det} B)^{1 / n} .
$$

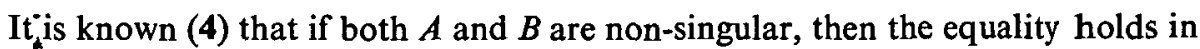
(1) if and only if $B=c A$ where $c$ is a positive number.

In this note we shall investigate the cases of equality in an extension of the result (1).

Theorem 1. For each $n \times n$ matrix $X$ and each integer $r, 1 \leqq r \leqq n$, let $d_{r}(X)$ denote the sum of all $r$-square principal subdeterminants of $X$. If $\bar{A}$ and $B$ are $n$-square positive semi-definite hermitian matrices and $0<q \leqq 1$, then

$$
d_{r}^{1 / r}\left((A+B)^{q}\right) \geqq 2^{q-1} d_{r}^{1 / r}\left(A^{q}\right)+2^{q-1} d_{r}^{1 / r}\left(B^{q}\right) .
$$

If $A$ and $B$ both have rank at least $r$ and if $q<1$, then equality holds in (2) if and only if $A=B$. If $q=1$ and $r>1$, then equality holds in (2) if and only if $B=c A$ for some $c>0$.

We shall deduce Theorem 1 from Theorem 2 below. In order to state Theorem 2 we introduce some notation and definitions.

By $C_{r}$ we denote the set of all $k$-tuples of non-negative reals with at least $r$ positive coordinates. If $f$ is a real valued function defined on $k$-tuples of reals then we say that

(i) $f$ is strictly $C_{r}$-concave if $f$ is concave on $C_{r}$ and for $x$ and $y$ in $C_{r}$ and for $0<\theta<1$, the equality $f(\theta x+(1-\theta) y)=\theta f(x)+(1-\theta) f(y)$ implies that $x$ is a positive multiple of $y, x \sim y$;

(ii) $f$ is $C_{r}$-positive means that $f(x)>0$ if and only if $x \in C_{r}$;

(iii) $f$ is strictly $C_{r}$-monotone if $f(x+u)>f(x)$ for $x$ in $C_{r}$ and for $u$ any non-zero $k$-tuple of non-negative reals.

Theorem 2. Let $A$ and $B$ be $n$-square positive semi-definite hermitian matrices with eigenvalues $0 \leqq \lambda_{1} \leqq \ldots \leqq \lambda_{n}$ and $0 \leqq \mu_{1} \leqq \ldots \leqq \mu_{n}$ respectively and let $0 \leqq \sigma_{1} \leqq \ldots \leqq \sigma_{n}$ denote the eigenvalues of $A+B$. Let $1 \leqq k \leqq n$ and assume

$\dagger$ The research of both authors was supported by the U.S. Air Force Office of Scientific Research under Grant AFOSR 698-67.

E.M.S. - X 
that $f(x)=f\left(x_{1}, \ldots, x_{k}\right)$ is symmetric concave and non-decreasing in each variable. Then

$$
2 f\left(\sigma_{1}, \ldots, \sigma_{k}\right) \geqq f\left(2 \lambda_{1}, \ldots, 2 \lambda_{k}\right)+f\left(2 \mu_{1}, \ldots, 2 \mu_{k}\right) .
$$

Assume that $1 \leqq r \leqq k$, and that $f$ is strictly $C_{r}$-monotone, strictly $C_{r}$-concave and $C_{r}$-positive. Moreover assume that $A$ and $B$ both have rank at least $n-k+r$. Then equality can hold in (3) if and only if there exists a unitary matrix $X$ such that

$$
\begin{aligned}
X^{*}(A+B) X & =\operatorname{diag}\left(\sigma_{1}, \ldots, \sigma_{n}\right), \\
X^{*} A X & =\operatorname{diag}\left(\lambda_{1}, \ldots, \lambda_{k}\right)+A_{n-k}, \\
X^{*} B X & =c \operatorname{diag}\left(\lambda_{1}, \ldots, \lambda_{k}\right)+B_{n-k}, c>0,
\end{aligned}
$$

$\mu_{i}=c \lambda_{i}, i=1, \ldots, k,\left(A_{n-k}\right.$ and $B_{n-k}$ are $(n-k)$-square matrices) and c satisfies

$$
2 f\left((1+c) \lambda_{1}, \ldots,(1+c) \lambda_{k}\right)=f\left(2 \lambda_{1}, \ldots, 2 \lambda_{k}\right)+f\left(2 c \lambda_{1}, \ldots, 2 c \lambda_{k}\right) .
$$

Proof. Let $x_{1}, \ldots, x_{n}$ be orthonormal eigenvectors of $A+B$ corresponding respectively to $\sigma_{1} \leqq \ldots \leqq \sigma_{n}$. Then

$$
\begin{aligned}
f\left(\sigma_{1}, \ldots, \sigma_{k}\right) & =f\left(\left((A+B) x_{1}, x_{1}\right), \ldots,\left((A+B) x_{k}, x_{k}\right)\right) \\
& =f\left(\left(A x_{1}, x_{1}\right)+\left(B x_{1}, x_{1}\right), \ldots,\left(A x_{k}, x_{k}\right)+\left(B x_{k}, x_{k}\right)\right) \\
& =f\left(\frac{\left(2 A x_{1}, x_{1}\right)+\left(2 B x_{1}, x_{1}\right)}{2}, \ldots, \frac{\left(2 A x_{k}, x_{k}\right)+\left(2 B x_{k}, x_{k}\right)}{2}\right) \\
& \geqq \frac{1}{2}\left[f\left(\left(2 A x_{1}, x_{1}\right), \ldots,\left(2 A x_{k}, x_{k}\right)\right)+f\left(\left(2 B x_{1}, x_{1}\right), \ldots,\left(2 B x_{k}, x_{k}\right)\right)\right] .
\end{aligned}
$$

Suppose that $u_{1}, \ldots, u_{n}$ are orthonormal eigenvectors of $A$ corresponding to $\lambda_{1}, \ldots, \lambda_{n}$ respectively. Then

$$
\left(A x_{i}, x_{i}\right)=\sum_{j=1}^{n}\left|\left(x_{i}, u_{j}\right)\right|^{2} \lambda_{j}
$$

Since the vectors $x_{1}, \ldots, x_{n}$ are also orthonormal it follows that the matrix $S$ whose $(i, j)$ element is $\left|\left(x_{i}, u_{j}\right)\right|^{2}$ is doubly stochastic. Thus by a theorem of Birkhoff $(1 ; 2$, p. 97) $S$ is a convex combination of permutation matrices

$$
S=\sum_{\sigma \in G} c_{\sigma} P_{\sigma}
$$

where $G$ is a subset of $S_{n}$, the permutation group of degree $n$. Let $\lambda$ denote the $n$-tuple $\left(\lambda_{1}, \ldots, \lambda_{n}\right)$. For each permutation $\sigma$ let $\lambda^{\sigma}$ denote $\left(\lambda_{\sigma(1)}, \ldots, \lambda_{\sigma(n)}\right)$ and for each $n$-tuple $x=\left(x_{1}, \ldots, x_{n}\right)$ let $x[k]=\left(x_{1}, \ldots, x_{k}\right)$. Then the concavity of $f$ implies that

$$
\begin{aligned}
f\left(\left(A x_{1}, x_{1}\right), \ldots,\left(A x_{k}, x_{k}\right)\right) & =f\left(\sum_{\sigma \in G} c_{\sigma} \lambda^{\sigma}[k]\right) \\
& \geqq \sum_{\sigma \in G} c_{\sigma} f\left(\lambda^{\sigma}[k]\right) .
\end{aligned}
$$


Since $f$ is symmetric and non-decreasing and $\lambda_{1} \leqq \ldots \leqq \lambda_{n}$,

$$
f\left(\lambda^{\sigma}[k]\right) \geqq f\left(\lambda_{1}, \ldots, \lambda_{k}\right) \text {. }
$$

Thus $f\left(\left(A x_{1}, x_{1}\right), \ldots,\left(A x_{k}, x_{k}\right)\right) \geqq f\left(\lambda_{1}, \ldots, \lambda_{k}\right)$. Similarly it follows that

Hence

$$
f\left(\left(B x_{1}, x_{1}\right), \ldots,\left(B x_{k}, x_{k}\right)\right) \geqq f\left(\mu_{1}, \ldots, \mu_{k}\right) \text {. }
$$

$$
f\left(\sigma_{1}, \ldots, \sigma_{k}\right) \geqq \frac{1}{2}\left[f\left(2 \lambda_{1}, \ldots, 2 \lambda_{k}\right)+f\left(2 \mu_{1}, \ldots, 2 \mu_{k}\right)\right] \text {. }
$$

This proves the inequality. Suppose that equality holds in (3), $f$ satisfies the given conditions, and $A$ and $B$ have rank at least $n-k+r$. Then at least $r$ of the inner products $\left(A x_{i}, x_{i}\right), i=1, \ldots, k$, must be positive and similarly at least $r$ of the inner products $\left(B x_{i}, x_{i}\right), i=1, \ldots, k$, must be positive. Now in (3) we proved the following result: let $H=\left(h_{i j}\right)$ be an $n$-square positive semidefinite hermitian matrix with eigenvalues $0 \leqq \gamma_{1} \leqq \ldots \leqq \gamma_{n}$; let $1 \leqq r \leqq k \leqq n$ and suppose that $f$ is a real valued function defined on the set of $k$-tuples of non-negative reals which is symmetric, concave and non-decreasing in each variable. Then for any set of $k$ orthonormal vectors $x_{1}, \ldots, x_{k}$

$$
f\left(\left(H x_{1}, x_{1}\right), \ldots,\left(H x_{k}, x_{k}\right)\right) \geqq f\left(\gamma_{1}, \ldots, \gamma_{k}\right) ;
$$

if $f$ is strictly $C_{r}$-monotone, strictly $C_{r}$-concave and $C_{r}$-positive and if at least $r$ of the inner products $\left(H x_{j}, x_{j}\right), j=1, \ldots, k$ are positive then the preceding inequality is equality if and only if

$$
H x_{j}=\gamma_{\phi(j)} x_{j}, j=1, \ldots, k
$$

for some permutation $\phi$ on $\{1, \ldots, k\}$, i.e., $x_{1}, \ldots, x_{k}$ is an orthonormal set of eigenvectors corresponding to $\gamma_{1}, \ldots, \gamma_{k}$ in some order. In view of this result and the strict $C_{r}$-concavity of $f$ we can conclude that

and

$$
\begin{aligned}
& A x_{j}=\lambda_{\phi(j)} x_{j}, \quad j=1, \ldots, k, \quad \phi \in S_{k}, \\
& B x_{j}=\mu_{\theta(j)} x_{j}, \quad j=1, \ldots, k, \quad \theta \in S_{k},
\end{aligned}
$$

$$
c \lambda[k]^{\phi}=\mu[k]^{\theta}, \quad c>0,
$$

where $\lambda=\left(\lambda_{1}, \ldots, \lambda_{n}\right), \mu=\left(\mu_{1}, \ldots, \mu_{n}\right)$. Let $\tau=\theta \phi^{-1}$ so that (5) becomes

$$
\mu_{\mathrm{r}(i)}=c \lambda_{i}, \quad i=1, \ldots, k \text {. }
$$

Since $\lambda_{1} \leqq \ldots \leqq \lambda_{k}$ and $\mu_{1} \leqq \ldots \leqq \mu_{k}$ we conclude from (6) that $\mu_{\tau(i)}=\mu_{i}$, $i=1, \ldots, k$, i.e., $c \lambda_{i}=\mu_{i}, i=1, \ldots, k$. But then

or

$$
\begin{aligned}
\mu_{\phi(i)} & =c \lambda_{\phi(i)} \\
& =\mu_{\theta(i)}
\end{aligned}
$$

However

$$
\mu_{\phi(i)}=\mu_{\theta(i)}, \quad i=1, \ldots, k .
$$

$$
\begin{aligned}
\sigma_{i} & =\left((A+B) x_{i}, x_{i}\right)=\left(A x_{i}, x_{i}\right)+\left(B x_{i}, x_{i}\right)=\lambda_{\phi(i)}+\mu_{\theta(i)} \\
& =\lambda_{\phi(i)}+\mu_{\phi(i)}=\lambda_{\phi(i)}+c \lambda_{\phi(i)}=(1+c) \lambda_{\phi(i)}, \quad i=1, \ldots, k .
\end{aligned}
$$


From $\sigma_{1} \leqq \ldots \leqq \sigma_{k}$, it follows that $\lambda_{\phi(i)}=\lambda_{i}, i=1, \ldots, k$, and hence

$$
\mu_{\theta(i)}=\mu_{i}=c \lambda_{i}, \quad i=1, \ldots, k .
$$

Thus $\sigma_{i}=(1+c) \lambda_{i}, i=1, \ldots, k$, and equality holds if and only if (4) holds.

Corollary. Let $A, B$, and $f$ satisfy the conditions of Theorem 2 and let $k=n$. If $f$ is homogeneous of degree $q \neq 0$ then

$$
f\left(\sigma_{1}, \ldots, \sigma_{n}\right) \geqq 2^{q-1} f\left(\lambda_{1}, \ldots, \lambda_{n}\right)+2^{q-1} f\left(\mu_{1}, \ldots, \mu_{n}\right) .
$$

If $A$ and $B$ both have rank at least $r$ then (7) is equality if and only if $B=c A$, $c>0$. If $q \neq 1$ then equality can hold if and only if $B=A$.

Proof. According to Theorem 2 there exists a unitary $X$ such that

and (from (4))

$$
\begin{aligned}
X^{*} A X & =\operatorname{diag}\left(\lambda_{1}, \ldots, \lambda_{n}\right), \\
X^{*} B X & =c \operatorname{diag}\left(\lambda_{1}, \ldots, \lambda_{n}\right), \quad c>0,
\end{aligned}
$$

$$
(1+c)^{q}=2^{q-1}\left(1+c^{q}\right)
$$

It is easy to check that for $q \neq 1, c=1$ is the only positive solution to (8). This completes the proof of the Corollary.

In (3) we show that if $E_{r}\left(y_{1}, \ldots, y_{n}\right)$ denotes the $r$ th elementary symmetric function of $y_{1}, \ldots, y_{n}$ and if $f\left(x_{1}, \ldots, x_{n}\right)=E_{r}^{1 / r}\left(x_{1}^{q}, \ldots, x_{n}^{q}\right)$ with $0<q \leqq 1$, then for $r>1$, or $r=1$ and $q<1, f$ is strictly $C_{r}$-concave. With this choice of $f$, Theorem 1 now follows from the Corollary.

\section{REFERENCES}

(1) G. Birkhoff, Tres observaciones sobre el algebra lineal, Univ. Nac. Tucuman Rev., Ser. A 5 (1946), 147-150.

(2) M. MARcus and H. MiNC, $A$ Survey of Matrix Theory and Matrix Inequalities (Prindle, Weber and Schmidt, Boston, Mass., 1964).

(3) W. R. GORDON and M. MARcus, An analysis of equality in certain matrix inequalities I, Pacific J. Math. 34 (1970), 407-413.

(4) L. MIRSKy, An Introduction to Linear Algebra (Oxford 1955), 419.

University of California, Santa Barbara

UNIVERSITY OF VICTORIA, VICTORIA B.C. 